EPJ Web of Conferences 60, 17013 (2013)

DOI: $10.1051 /$ epjconf $/ 20136017013$

(C) Owned by the authors, published by EDP Sciences, 2013

\title{
Search for Heavy Resonances in Leptonic Final States with CMS
}

\author{
John Stupak III ${ }^{1, a}$ \\ On Behalf of the CMS Collaboration \\ ${ }^{1}$ Department of Chemistry and Physics, Purdue University Calumet, Hammond, IN 46323
}

\begin{abstract}
Recent searches are presented for heavy resonances, such as $Z^{\prime}, W^{\prime}$, and leptoquarks, in leptonic final states. LHC collisions at $\sqrt{s}=8 \mathrm{TeV}$ are analyzed with the CMS detector. No deviations from Standard Model expectations are observed. Cross section and mass limits are established for a variety of new physics scenarios, which in many cases represent the most stringent limits in existence.
\end{abstract}

\section{Introduction}

The Standard Model (SM) is a gauge theory possessing $\mathrm{SU}(3)_{\mathrm{C}} \times \mathrm{SU}(2)_{\mathrm{L}} \times \mathrm{U}(1)_{\mathrm{Y}}$ symmetry. A common approach to explain Beyond the Standard Model (BSM) physics is to extend the SM gauge symmetry to include an additional gauge symmetry $G$, so that the postulated symmetry is $\mathrm{SU}(3)_{\mathrm{C}} \times \mathrm{SU}(2)_{\mathrm{L}} \times \mathrm{U}(1)_{\mathrm{Y}} \times G$. Simple examples of such extensions include $G=\mathrm{U}(1)^{\prime}[1,2]$ and $G=\mathrm{SU}(2)^{\prime}[1,3-$ $6]$. In the former case, such an extension requires introduction of an additional electrically-neutral gauge boson associated with the $\mathrm{U}(1)^{\prime}$ symmetry. In the latter case, such an extension requires introduction of two additional electrically-charged and one additional electrically-neutral gauge bosons associated with the $\mathrm{SU}(2)^{\prime}$ symmetry. We refer to these additional charged and neutral gauge bosons generically as $W^{\prime}$ and $Z^{\prime}$ bosons, respectively.

An alternative approach to explain BSM Physics is to embed the SM within a larger symmetry group. This is the approach taken in Grand Unified Theories (GUTs). Common GUT symmetry groups which contain the $\mathrm{SU}(3)_{\mathrm{C}} \times \mathrm{SU}(2)_{\mathrm{L}} \times \mathrm{U}(1)_{\mathrm{Y}}$ of the $\mathrm{SM}$ include $\mathrm{SU}(5)$ [7], SU(15) [8], SO(10) [9], and $\mathrm{E}_{6}$ [10]. This class of models generally predicts additional charged and neutral gauge bosons. In addition, since quarks and leptons are placed together within a multiplet, leptoquarks [1116] are generally predicted as well. Leptoquarks are color triplet gauge bosons which carry both lepton and baryon number, and therefore decay to a lepton-quark pair. Constraints based on searches for flavor changing neutral current and lepton flavor violation imply that any leptoquark inter-generational coupling must be small $[17,18]$. Therefore, it is typically assumed that leptoquarks couple only to a single generation of fermions.

A variety of other classes of models also predict additional gauge bosons and/or leptoquarks, including composite models [19, 20], R-parity violating SUSY [21], Lit-

\footnotetext{
ae-mail: john.stupak@cern.ch
}

tle Higgs models [22, 23], Technicolor [24, 25], and models with extra dimensions [26, 27]. Leptonic decays of these heavy resonances offer a promising opportunity for discovery. The lepton $(e, \mu)$ may be used for triggering and background rejection, and in many scenarios the leptonic decays can have a large branching ratio.

\section{The Searches}

The searches presented here are based on $\sqrt{s}=8 \mathrm{TeV}$ data collected with the CMS detector [28]. Unless otherwise noted, the data analyzed corresponds to the entire 2012 dataset, and was collected with single or dilepton triggers. For each analysis, all possible sources of systematic uncertainty are carefully considered and treated as nuisance parameters during limit setting.

\subsection{Right-Handed Charged Gauge Boson}

The Left-Right Symmetric model [3-6] predicts heavy right-handed (RH) neutrinos $N_{\ell}$ and charged gauge bosons $W_{R}$ with couplings to $\mathrm{RH}$ fermions. If produced at the LHC, the $W_{R}$ could decay via $W_{R} \rightarrow \ell N_{\ell} \rightarrow \ell \ell W_{R}^{*} \rightarrow$ $\ell \ell j j$. CMS performed a search [29] for such a cascade decay in the first $3.6 \mathrm{fb}^{-1}$ of the 2012 dataset. The strategy of the analysis is to select events consistent with the $\ell \ell j j$ final state and search for a resonance in the $m_{\ell \ell j j}$ distribution.

Events are required to contain at least two high- $p_{T}$, same-flavor, isolated leptons, where the leading (subleading) lepton $p_{T}$ exceeds 60 (40) $\mathrm{GeV}$, and at least two jets with $p_{T}$ in excess of $40 \mathrm{GeV}$. To reduce the contribution from $Z+$ jets events, the dilepton invariant mass $m_{\ell \ell}$ is required to exceed $200 \mathrm{GeV}$.

The dominant backgrounds, $t \bar{t}$ and $Z+$ jets, are modelled using data-driven techniques. The $t \bar{t}$ shape and normalization are derived from $e \mu j j$ events. The $Z+j$ ets shape is modelled with MadGraph [30], and the normalization is extracted from data in a window around the $Z$ 


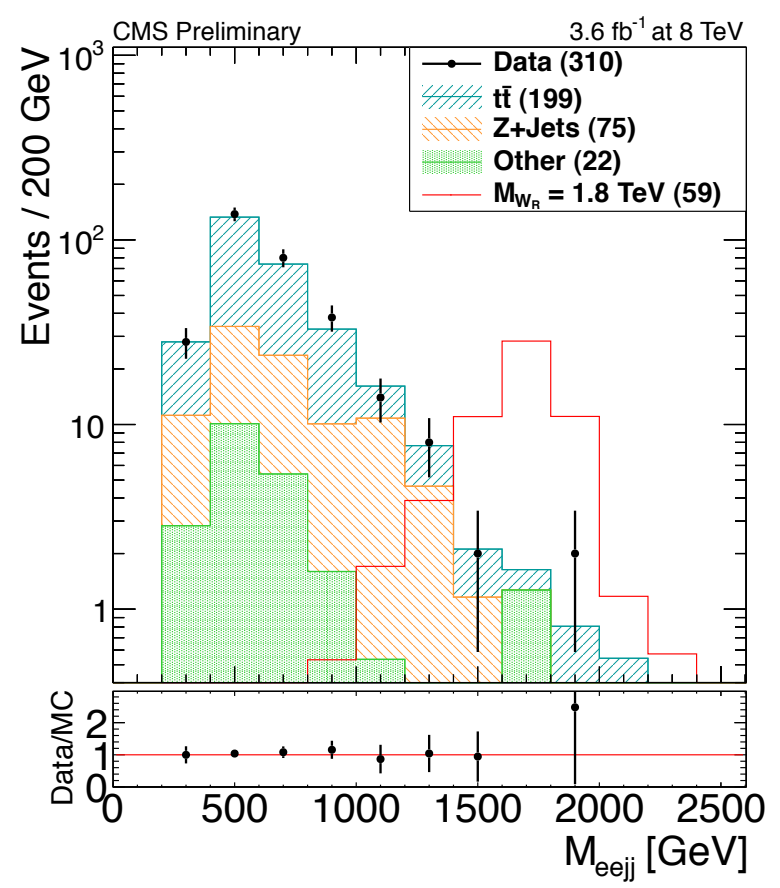

Figure 1. The expected and observed $m_{e e j j}$ distributions for selected events in the electron channel. SM backgrounds are represented by stacked, hatched histograms. Data is represented by circles with error bars. A $1.8 \mathrm{TeV} W_{R}$ signal is represented by the red histogram. Good agreement is found between data and the SM background.

mass. The background from QCD, although minor, is also modelled with a data-driven technique. All other backgrounds are modelled with MC.

The expected and observed $m_{e e j j}$ distributions for selected events in the electron channel are shown in Figure 1. A similar distribution was found in the muon channel. No deviation from the SM background prediction is observed. As a result, 95\% Confidence Level (CL) upper limits on $\sigma\left(p p \rightarrow W_{R}\right) \times \mathrm{BR}\left(W_{R} \rightarrow[e e+\mu \mu+\tau \tau] j j\right)$ are determined. These limits are then reinterpreted in terms of the $N_{\ell}$ and $W_{R}$ masses, and shown in Figure 2. For intermediate $N_{\ell}$ masses, a $W_{R}$ with mass below $2.8 \mathrm{TeV}$ is excluded at $95 \% \mathrm{CL}$.

\subsection{Charged Gauge Boson}

Many models predict a heavy $W^{\prime}$ boson. CMS performed a search [31] for leptonic $W^{\prime}$ decays. The signal would appear as a Jacobian peak in the tail of the SM transverse mass distribution, where transverse mass is defined as $m_{T}=\sqrt{2 \cdot p_{T}^{\ell} \cdot \mathbb{E}_{T} \cdot\left[1-\cos \Delta \phi\left(\ell, \mathbb{E}_{T}\right)\right]}$.

Events are selected with exactly one high- $p_{T}$, isolated lepton. The electron (muon) $p_{T}$ is required to exceed 100 (45) GeV. In signal events, the lepton $p_{T}$ and $\mathbb{E}_{T}$ are expected to be roughly equal in magnitude and back-to-back in $\phi$. Thus, cuts of $0.4<p_{T}^{\ell} / \mathbb{E}_{T}<1.5$ and $\Delta \phi\left(\ell, \mathbb{E}_{T}\right)>$ $0.8 \pi$ are applied.

The dominant background is SM $W+$ jets production. The shape of the $W+$ jets background is modelled at Lead-

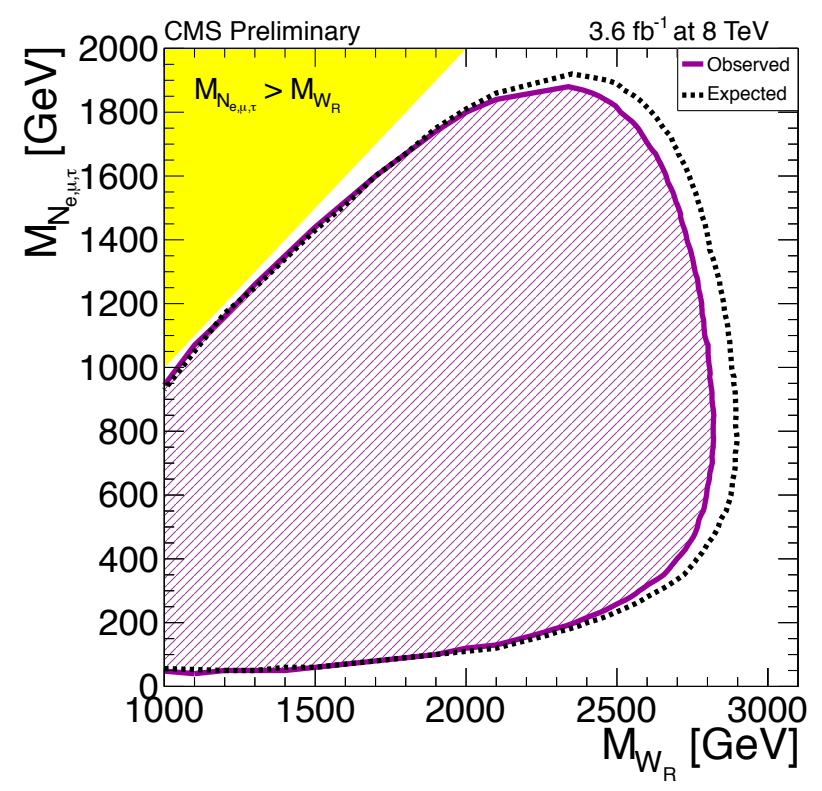

Figure 2. The expected and observed 95\% CL limits, assuming degenerate $N_{\ell}$, for the combination of the electron and muon channels. The expected (observed) limit is represented by a solid purple (dashed black) line.

ing Order (LO) with Pythia [32]. This shape is then reweighted using an $m_{T}$ dependent Next-to-Leading Order (NLO) k-factor. Finally, the $W+$ jets background expectation is normalized to the Next-to-Next-to-Leading Order (NNLO) cross section. Other backgrounds are modelled with MC as well. In order to reduce statistical fluctuations in the tail of the expected $m_{T}$ distribution, an empirical function is fit to the total background expectation:

$$
\frac{a}{\left(m_{T}^{3}+b m_{T}+c\right)^{d}}
$$

The expected and observed $m_{T}$ distributions for selected events in the muon channel are shown in Figure 3. A similar distribution was found in the electron channel. The observed distribution is consistent with SM background expectations. 95\% CL limits are established for a number of benchmark models. The upper bound on $\sigma \times \mathrm{BR}$ from the combination of the electron and muon channels is shown in Figure 4, along with the theoretical predictions for a $W^{\prime}$ in the Sequential Standard Model (SSM) [1] and the first even Kaluza-Klein excitation $W_{K K}^{2}$ in splitUED [26, 27] for two different bulk mass parameters $\mu$. Limits are also established in the context of the SSM considering the potential for constructive or destructive interference between a $W^{\prime}$ and the SM $W$, and in the context of a Helicity Non-Conserving (HNC) contact interaction model [33]. The 95\% CL mass limits for these models are summarized in Table 1.

\subsection{Neutral Gauge Boson}

An additional neutral $Z^{\prime}$ gauge boson $[1,2]$ is predicted by many BSM theories, which could decay to an oppositesign, same-flavor lepton pair. The strategy used in this 

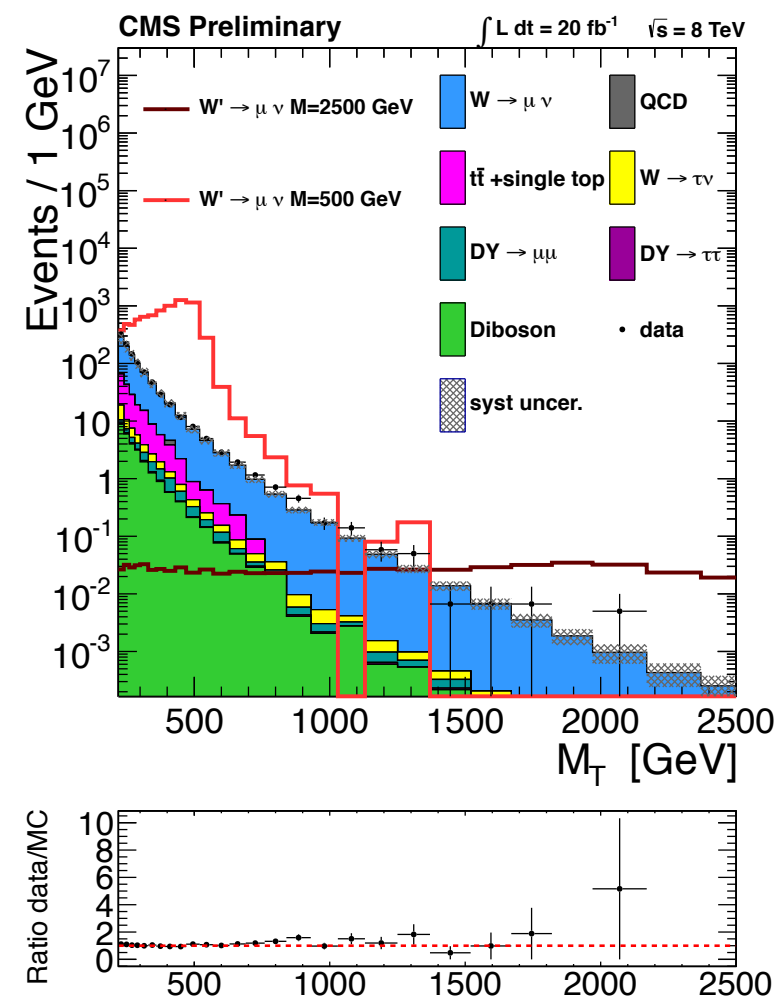

Figure 3. The expected and observed $m_{T}$ distributions for selected events in the muon channel. SM backgrounds are represented by stacked, filled histograms. Data is represented by circles with error bars. A $500 \mathrm{GeV}(2.5 \mathrm{TeV}) W^{\prime}$ signal is represented by the red (maroon) histogram. Good agreement is found between data and the SM background expectation.

Table 1. Observed 95\% CL limits for a variety of models.

\begin{tabular}{|c|c|c|}
\hline Model & Channel & Observed Limit \\
\hline \hline SSM & combined & $m_{W^{\prime}}<3.35 \mathrm{TeV}$ \\
\hline SSMO & combined & $m_{W^{\prime}}<3.60 \mathrm{TeV}$ \\
SSMS & combined & $m_{W^{\prime}}<3.10 \mathrm{TeV}$ \\
\hline$W_{K K}^{2}(\mu=0.05 \mathrm{TeV})$ & combined & $m_{W_{K K}^{2}}<1.7 \mathrm{TeV}$ \\
$W_{K K}^{2}(\mu=10.0 \mathrm{TeV})$ & combined & $m_{W_{K K}^{2}}<3.7 \mathrm{TeV}$ \\
\hline HNC CI & $e$ & $\Lambda<13.0 \mathrm{TeV}$ \\
HNC CI & $\mu$ & $\Lambda<10.9 \mathrm{TeV}$ \\
\hline
\end{tabular}

analysis [34] is to select events consistent with such a decay and search for a resonance in the SM $m_{\ell \ell}$ tail.

Events are selected with exactly two same-flavor, high$p_{T}$, isolated leptons. Selected electrons (muons) are required to have $p_{T}>35$ (40) GeV. In the muon channel, where charge misidentification typically implies momentum mismeasurement, an opposite sign requirement is applied.

The dominant background in this analysis is SM $Z+$ jets production. The $Z+$ jets background is modelled at NLO with Powheg [35], and normalized to the NNLO cross section. The background from QCD events is modelled with a data-driven "fake rate" method. All other backgrounds are modelled with MC. The total background is normalized to data in a window around the $Z$ mass. In

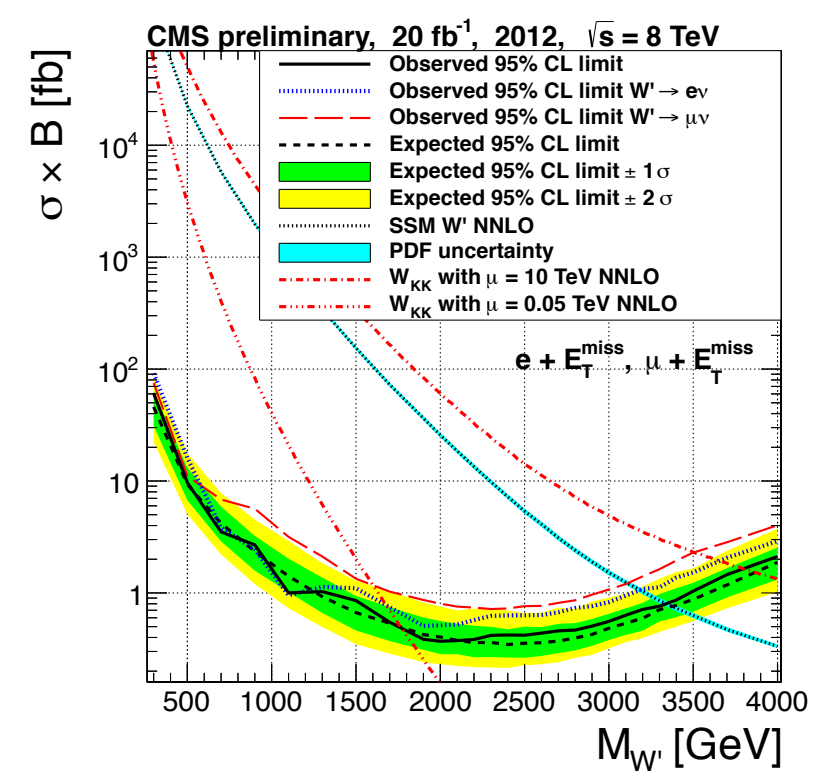

Figure 4. The expected and observed 95\% CL limits for the combination of the electron and muon channels. The expected (observed) limit is represented by a dashed (solid) black line, with $\pm 1 \sigma$ and $\pm 2 \sigma$ uncertainty bands shown in green and yellow respectively. The NNLO cross section for $W^{\prime}$ production in the SSM is represented by the dotted black line with a cyan PDF uncertainty band. The NNLO cross sections for $W_{K K}^{2}$ production with two choices of the bulk mass parameter $\mu$ are represented by the dashed-dotted red lines.

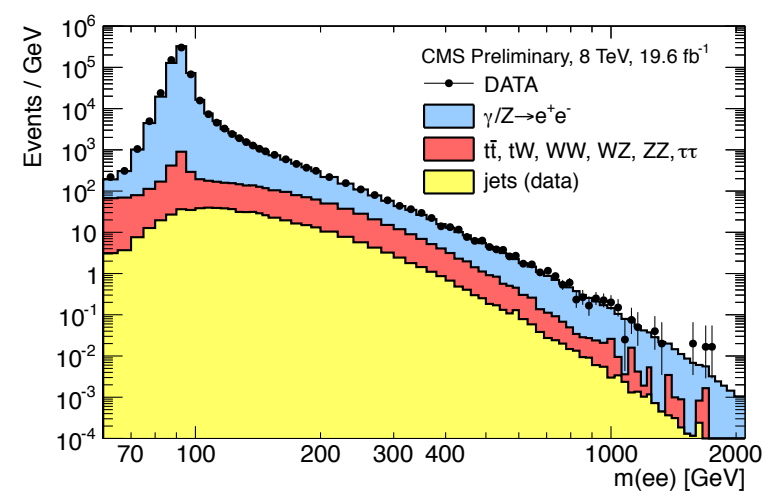

Figure 5. The expected and observed $m_{e e}$ distributions for selected events in the electron channel. SM backgrounds are represented by stacked, filled histograms. Data is represented by circles with error bars. Good agreement is found between data and the SM background.

order to reduce statistical fluctuations in the tail of the background $m_{\ell \ell}$ distribution, the final background expectation is obtained from a fit to the empirical function:

$$
m_{\ell \ell}^{k} \mathrm{e}^{\alpha m_{\ell \ell}+\beta m_{\ell \ell}^{2}}
$$

The expected and observed $m_{\ell \ell}$ distributions for selected events in the electron channel are shown in Figure 5. A similar distribution is found in the muon channel. In both channels, good agreement is found between 


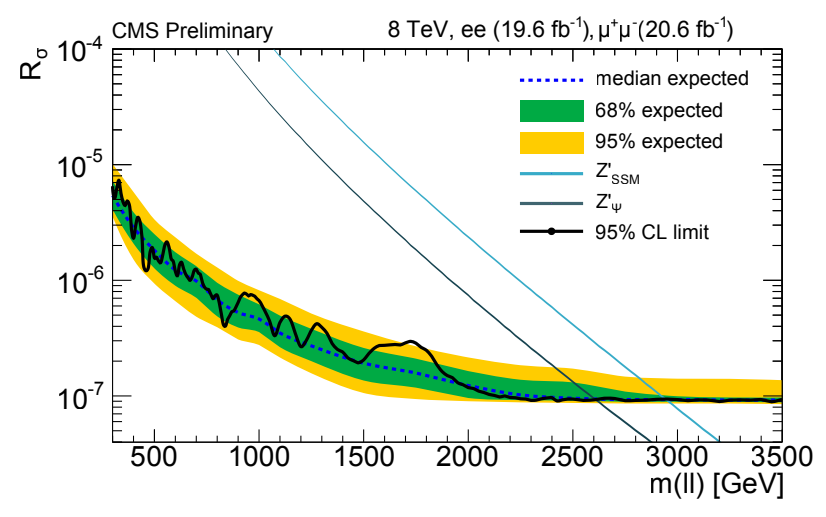

Figure 6. The expected and observed 95\% CL limits for the combination of the electron and muon channels. The expected (observed) limit is represented by a dashed blue (solid black) line, with $\pm 1 \sigma$ and $\pm 2 \sigma$ uncertainty bands shown in green and yellow respectively. The cross section for $Z^{\prime}$ production within the SSM (a superstring-inspired GUT) is represented by the light (dark) blue line.

the observed and expected background $m_{\ell \ell}$ distributions. Therefore, the $95 \%$ CL upper bound on

$$
R_{\sigma}=\frac{\sigma\left(p p \rightarrow Z^{\prime}+X \rightarrow \ell \ell+X\right)}{\sigma(p p \rightarrow Z+X \rightarrow \ell \ell+X)}
$$

are established, and shown in Figure 6. In this ratio many systematic uncertainties cancel, allowing stronger limits to be set than would otherwise be possible. Within the context of the SSM (a superstring-inspired GUT [2]), a $Z^{\prime}$ is excluded at 95\% CL with a mass below $2.96(2.60) \mathrm{TeV}$. The SSM limit is the strongest in existence.

\subsection{Second Generation Scalar Leptoquark Pair-Production}

A search [36] was performed by CMS for pair-production of second generation scalar leptoquarks. Second generation leptoquarks decay to a light quark and a muon (neutrino) with a model-dependent branching ratio $\beta(1-\beta)$. Therefore, the analysis is performed in two distinct channels, where either one or both leptoquarks decay to a muon and a light quark, giving $\mu v q q^{\prime}$ and $\mu \mu q q$ final states, respectively.

In both channels, events are pre-selected by requiring at least two jets, where the $p_{T}$ of the leading (sub-leading) jet is required to exceed $125(45) \mathrm{GeV}$, and $S_{T}$ (the scalar sum of the $p_{T}$ all four leptoquark decay products) in excess of $300 \mathrm{GeV}$. Muons are required to be isolated and high- $p_{T}\left(p_{T}>45 \mathrm{GeV}\right)$. In the single muon channel, an electron veto is applied. The $\mathbb{E}_{T}$ is required to be large $\left(\mathbb{E}_{T}>50 \mathrm{GeV}\right)$, and well separated in $\phi$ from the muon and leading jet. Finally, the transverse mass is required to exceed $50 \mathrm{GeV}$. In the dimuon channel, the dimuon invariant mass is required to exceed $50 \mathrm{GeV}$.

A mass-dependent event selection is then performed. For both channels, three kinematic variables are selected which discriminate strongly between signal and background. Mass-dependent cut thresholds are chosen for

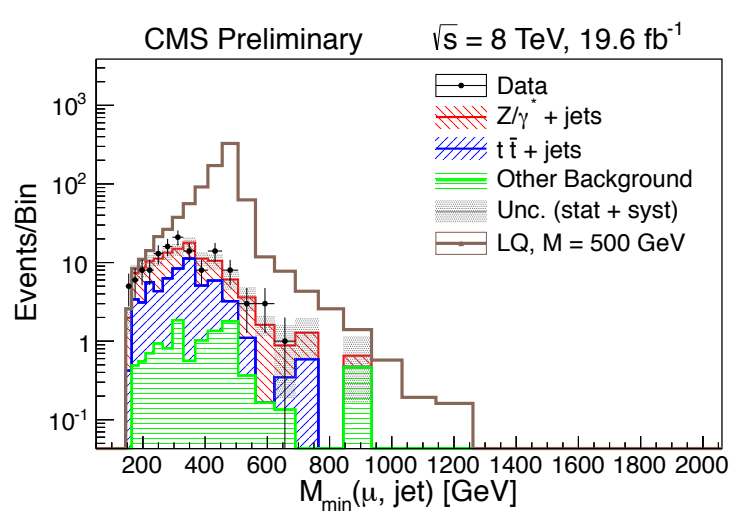

Figure 7. The expected and observed $m_{\min }(\mu$, jet) distributions for selected events in the dimuon channel for a $500 \mathrm{GeV}$ leptoquark mass hypothesis. SM backgrounds are represented by stacked, hatched histograms. Data is represented by circles with error bars. A $500 \mathrm{GeV}$ leptoquark signal is represented by the brown histogram. Good agreement is found between data and the SM background expectation.

these variables which optimize the signal sensitivity for each signal mass hypothesis. In the single muon channel, the kinematic variables used are $S_{T}, m_{T}$, and $m(\mu$, jet) (the invariant mass of the muon-jet pair which minimizes the difference in the transverse mass between the muon-jet and $\mathbb{E}_{T}$-jet pairs). In the dimuon channel, the kinematic variables used are $S_{T}, m_{\mu \mu}$, and $m_{\min }(\mu$, jet) (the smaller of the two muon-jet invariant masses which minimizes the $L Q-\overline{L Q}$ invariant mass difference).

The dominant background in the single muon channel is $W+$ jets production. The shape of the $W+$ jets background is taken from MadGraph, and normalized to the pre-selected data in a window around the $W$ transverse mass peak. The dominant backgrounds in the dimuon channel are $Z+$ jets and $t \bar{t}$. The $Z+$ jets shape is taken from MadGraph, and normalized to the pre-selected data in a window around the $Z$ mass. Both the shape and normalization of the $t \bar{t}$ background are taken from data events with an electron and a muon.

The expected and observed $m_{\min }(\mu$, jet) distributions for selected events in the dimuon channel are shown in Figure 7. In both channels, good agreement is found between data and background expectation. Therefore, 95\% CL cross section limits are established for each channel. The two channels are then combined, and the limits are interpreted in the branching ratio $\beta$ versus leptoquark mass plane. For $\beta=0.5$ (1), leptoquark masses below 785 (1070) $\mathrm{GeV}$ are excluded at 95\% CL. These are the strongest limits in existence.

\section{Conclusion}

Several searches have been presented for heavy resonances decaying to leptonic final states. No deviations form the SM background expectation are observed. As a result, cross section and mass limits are established for a variety of potential new physics scenarios. 


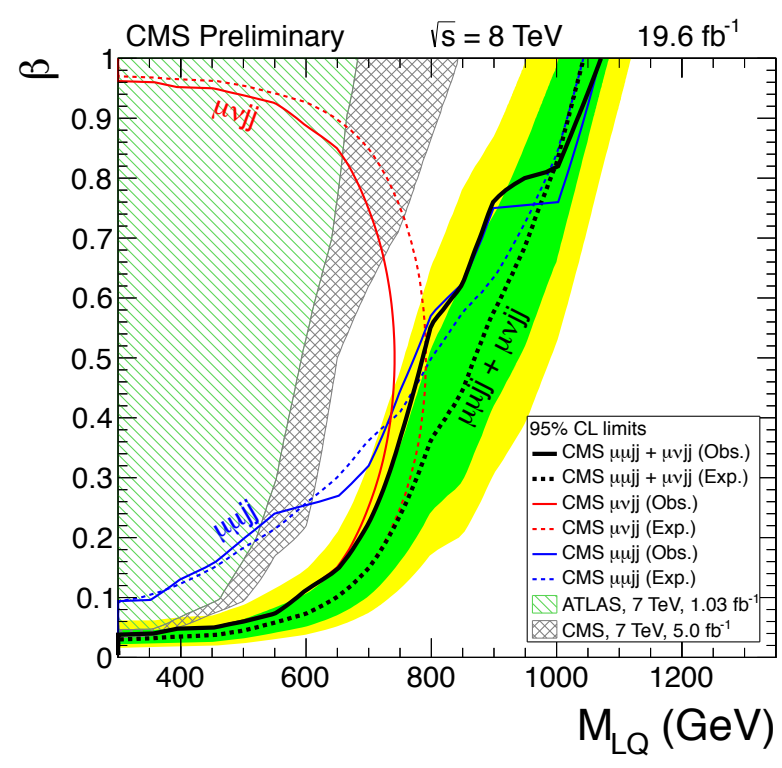

Figure 8. The expected and observed 95\% CL limits for the single muon channel (red), dimuon channel (blue), and the combination of the two channels (black), in the branching ratio $\beta$ versus leptoquark mass plane. The expected (observed) limits are represented by dotted (solid) lines. The $\pm 1 \sigma$ and $\pm 2 \sigma$ uncertainty bands for the combined limit are shown in green and yellow, respectively. The previous ATLAS (CMS) limit based on $7 \mathrm{TeV}$ data is represented by the hatched green (black) regions.

\section{References}

[1] G. Altarelli, B. Mele, M. Ruiz-Altaba, Zeitschrift für Physik C Particles and Fields 45, 109 (1989)

[2] A. Leike, Phys.Rept. 317, 143 (1999), hep-ph/9805494

[3] J.C. Pati, A. Salam, Phys. Rev. D 10, 275 (1974)

[4] R.N. Mohapatra, J.C. Pati, Phys. Rev. D 11, 2558 (1975)

[5] G. Senjanovic, R.N. Mohapatra, Phys. Rev. D 12, 1502 (1975)

[6] W.Y. Keung, G. Senjanović, Phys. Rev. Lett. 50, 1427 (1983)

[7] H. Georgi, S.L. Glashow, Phys. Rev. Lett. 32, 438 (1974)

[8] P.H. Frampton, B.H. Lee, Phys. Rev. Lett. 64, 619 (1990)

[9] J.C. Pati, A. Salam, Phys. Rev. D 10, 275 (1974)

[10] E. Witten, Nuclear Physics B 258, 75 (1985)

[11] W. Buchmüller, R. Rückl, D. Wyle, Physics Letters B 191, 442 (1987)

[12] S. Dimopoulos, L. Susskind, Nuclear Physics B 191, 370 (1981)

[13] S. Dimopoulos, Nuclear Physics B 168, 69 (1980)
[14] E. Eichten, K. Lane, Physics Letters B 90, 125 (1980)

[15] V. Angelopoulos, J. Ellis, H. Kowalski, D. Nanopoulos, N. Tracas, F. Zwirner, Nuclear Physics B 292, 59 (1987)

[16] J.L. Hewett, T.G. Rizzo, Phys.Rev. D56, 5709 (1997), hep-ph/9703337

[17] W. Buchmuller, D. Wyler, Physics Letters B 177, 377 (1986)

[18] O. Shanker, Nuclear Physics B 204, 375 (1982)

[19] B. Schrempp, F. Schrempp, Physics Letters B 153, 101 (1985)

[20] H. Harari, Phys. Letters B 86, 87 (1979)

[21] R. Barbier, C. Bérat, M. Besançon, M. Chemtob, A. Deandrea, E. Dudas, P. Fayet, S. Lavignac, G. Moreau, E. Perez et al., Physics Reports 420, 1 (2005)

[22] N. Arkani-Hamed, A.G. Cohen, H. Georgi, Physics Letters B 513, 232 (2001)

[23] N. Arkani-Hamed, A.G. Cohen, E. Katz, A.E. Nelson, T. Gregoire, J.G. Wacker, Journal of High Energy Physics 2002, 021 (2002)

[24] E. Eichten, K. Lane, Physics Letters B 90, 125 (1980)

[25] S. Dimopoulos, L. Susskind, Nuclear Physics B 155, 237 (1979)

[26] C.R. Chen, M.M. Nojiri, S.C. Park, J. Shu, M. Takeuchi, Journal of High Energy Physics 2009, 078 (2009)

[27] K. Kong, S. Chan Park, T. Rizzo, Journal of High Energy Physics 2010, 1 (2010)

[28] CMS Collaboration, Journal of Instrumentation 3, S08004 (2008)

[29] CMS Physics Analysis Summary CMSPAS-EXO-12-017, CERN, Geneva (2012), http: //cds. cern.ch/record/1460445

[30] J. Alwall, M. Herquet, F. Maltoni, O. Mattelaer, T. Stelzer, JHEP 1106, 128 (2011), 1106.0522

[31] CMS Physics Analysis Summary CMSPAS-EXO-12-060, CERN, Geneva (2013), http: //cds . cern. ch/record/1522476

[32] T. Sjostrand, S. Mrenna, P.Z. Skands, Comput.Phys.Commun. 178, 852 (2008), 0710. 3820

[33] K. Lane, F. Paige, T. Skwarnicki, W. Womersley, Phys.Rept. 278, 291 (1997), hep-ph/9412280

[34] CMS Physics Analysis Summary CMSPAS-EXO-12-061, CERN, Geneva (2013), http: //cds. cern.ch/record/1519132

[35] S. Frixione, P. Nason, C. Oleari, JHEP 0711, 070 (2007), 0709. 2092

[36] CMS Physics Analysis Summary CMSPAS-EXO-12-042, CERN, Geneva (2013), http: //cds. cern. ch/record/1542374 